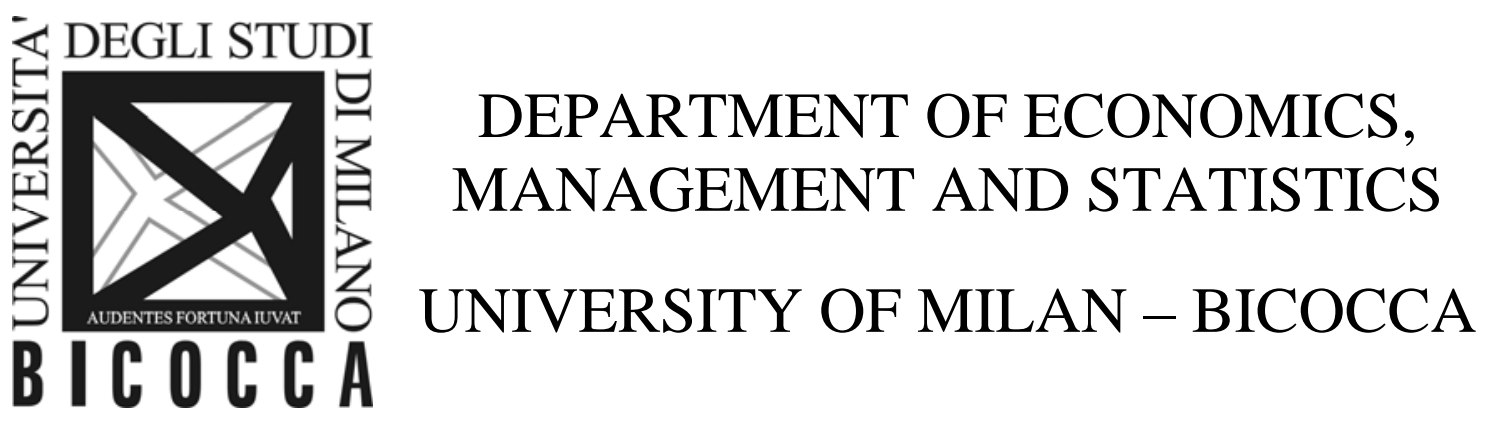

DEMS WORKING PAPER SERIES

Nonparametric tests for event studies under cross-sectional dependence

Matteo Pelagatti

No. 244 - May 2013

Dipartimento di Economia, Metodi Quantitativi e Strategie di Impresa Università degli Studi di Milano - Bicocca

http://dems.unimib.it/ 


\title{
Nonparametric tests for event studies under cross-sectional dependence
}

\author{
Matteo M. Pelagatti* \\ Department of Economics, Management and Statistics \\ Università degli Studi di Milano-Bicocca
}

May 2013

\begin{abstract}
We propose three nonparametric tests for the null of no eventinduced shifts in the distribution of stock returns. One test is the natural extension of the popular Corrado rank test to the case of crosssectionally dependent returns, while the other two are based on new ideas. Unfortunately only for one of these tests a solid theory for approximating the distribution of the statistic can be derived, but some simulation experiments confirm that normality is a good approximation also for the other two. The new tests are compared to a widely used parametric test (Patell) through simulation experiments and are shown to compare favourably in terms of power. Simulation results are based on bootstrapping daily stock returns from the S\&P100 and NASDAQ indexes.

Keywords: Rank test, Event study, Abnormal returns, Cross-sectional dependence

JEL: G14, C12, C14

*Email: matteo.pelagatti@unimib.it
\end{abstract}




\section{Introduction}

The use of rank-based nonparametric tests for event studies has a long history in the financial literature (McConnell and Muscarella, 1985; Corrado, 1989; Lummer and McConnell, 1989; Zivney and Thompson, 1989; Corrado and Zivney, 1992). The need for nonparametric (distribution free) tests is mainly due to the non-Gaussian nature of financial returns, which tend to be highly leptokurtic and often skewed. Indeed, Gaussian parametric tests such as the Patell T-test (Patell, 1976; Dodd and Warner, 1983) and the BMP T-test (Boehmer et al., 1991; Sanders and Robins, 1991) are often found oversized for real-world stock returns (Corrado and Truong, 2008; Bartholdy et al., 2007).

However, the existing nonparametric tests have been designed to work under the assumption of cross-sectional independence of abnormal returns, which does not hold when a panel of contemporaneous time series with common event date is analysed.

In this paper we propose a novel nonparametric test under the null hypothesis of no shift in the event-window returns and derive its asymptotic distribution. This test is valid under cross sectional dependence, for any length of the event window and any number of stocks in the analysis. Differently from existing nonparametric tests, the asymptotic approximation improves as the estimation window length increases regardless of the event window size. This implies that, under the typical event study set-up, the asymptotic approximation is extremely accurate.

The performance of this test is compared with the standard parametric Patell test, whose distribution is known only under the hypothesis of joint normality of the asset returns. The test is also compared with other two nonparametric tests, for which an exact asymptotic theory cannot be derived for the non-existence of central limit theorems under general crosssectional dependence. One of these two nonparametric tests is the natural generalisation of the test of Corrado (1989) to the case of cross-sectional dependence.

A simulation study based on bootstrapping daily returns from the S\&P100 and NASDAQ constituents shows that the first test we propose has very good size properties under any of the considered set-ups, but tends to have lower power than the other two nonparametric tests most of the times. The generalisation of the Corrado test has poor size properties when the number of stocks in the analysis is small, while the third test we propose tends to 
have both good size and high power. Despite the lack of central limit theorems under general dependence, the three comparison test statistics seem to converge in distribution to a normal random variable as the number of stocks diverges.

The paper is organised as follows: Section 2 introduces the new test and presents its asymptotic theory, Section 3 illustrates the three comparison tests which lack a solid asymptotic theory, Section 4 contains the simulation experiment and Section 5 concludes. The proof of the theorem in Section 2 can be found in the Appendix.

\section{A nonparametric test and its approximate dis- tribution}

Denote the estimation window by $\Omega_{0}$ and the event window by $\Omega_{1}$ and the relative cardinalities (i.e., the number of observations therein) by $n_{0}$ and $n_{1}$, and set $n=n_{0}+n_{1}$. Generally, in applications $n_{0} \approx 250$ and $n_{1} \approx 5$ daily returns. Suppose that the returns on the $i$-th stock, $y_{i t}$, for $i \in\{1, \ldots, m\}$ and $t \in \Omega_{0}$, are well represented by the linear model

$$
y_{i t}=\boldsymbol{x}_{i t}^{\top} \boldsymbol{\beta}+\varepsilon_{i t},
$$

where $\varepsilon_{t}$ is an i.i.d. process and $\boldsymbol{x}_{i t}$ is a vector of regressors, which usually include a constant and one or more market indexes.

The null hypothesis to test in event studies is that the model (1) holds for $t \in \Omega_{1}$ with the same distributional assumptions on the error term $\varepsilon_{i t}$. Under the alternative hypothesis the regression errors undergo a positive or negative shift in the location parameter (median or mean if it exists).

Now, define $\Omega=\Omega_{0} \cup \Omega_{1}$ the complete sample and let $\hat{\boldsymbol{\beta}}$ be a consistent estimator of the coefficients $\boldsymbol{\beta}$ computed only on the estimation window $\Omega_{0}$. The regression residuals are, then, given by

$$
\hat{\varepsilon}_{i t}=y_{i t}-\boldsymbol{x}_{i t}^{\top} \hat{\boldsymbol{\beta}}, \quad \text { for } t \in \Omega,
$$

and, since the scale parameter (standard deviation if it exists) of each $\hat{\varepsilon}_{i t}$ may vary over $i$, let us define the standardized residuals as

$$
e_{i t}=\frac{\hat{\varepsilon}_{i t}}{\hat{\sigma}_{i}},
$$


where $\hat{\sigma}_{i}$ is a consistent estimator for the scale parameter $\sigma_{i}$ of the regression error $\varepsilon_{i t}{ }^{1}$

Take the cross-sectional sums of the standardized residuals, $e_{t}=\sum_{i=1}^{m} e_{i t}$, and their ranks for the complete sample $\Omega$,

$$
R_{n t}=\sum_{k \in \Omega} \mathbb{1}_{\left(-\infty, e_{t}\right]}\left(e_{k}\right),
$$

with $\mathbb{1}_{A}(\cdot)$ indicator function for the set $A$. The first test statistic we propose is

$$
Z_{n}=\frac{1}{\sqrt{n_{1}}} \sum_{t \in \Omega_{1}} \Phi^{-1}\left(\frac{R_{n t}}{n+1}\right),
$$

where $\Phi^{-1}(\cdot)$ is the standard normal quantile function. Under the null hypothesis supplemented with mild regularity conditions (see below) $Z_{n}$ is approximately distributed as a standard normal, whatever the distribution of $\varepsilon_{i t}$ and $\boldsymbol{x}_{i t}$.

Theorem 1. Assume that (1) holds with i.i.d. vector of error terms $\varepsilon_{t}=$ $\left(\varepsilon_{1 t}, \ldots, \varepsilon_{m t}\right)^{\top}$ and

1. the distribution functions of $\varepsilon_{t}=\sum_{i=1}^{m} \varepsilon_{i t} / \sigma_{i}, F(\cdot)$, is absolutely continuous with absolutely continuous density $f(\cdot)$ and support $\Theta$;

2. $I_{0}(f)=\int_{\Theta}\left[f^{\prime}(x) / f(x)\right]^{2} f(x) \mathrm{d} x<\infty$ (finite information for location);

3. $\hat{\sigma}_{i}=\sigma_{i}+O_{p}\left(n^{-1 / 2}\right)$, for $i=1, \ldots, m$;

4. $\max _{1 \leq t \leq n_{0}} x_{j i t}^{2} /\left(\sum_{t \in \Omega_{0}} x_{j i t}^{2}\right) \rightarrow 0$ as $n_{0}$ diverges, for $j=1, \ldots, k, i=1, \ldots, m$;

5. $\sqrt{\sum_{t \in \Omega_{0}} x_{j i t}^{2}}\left(\hat{\beta}_{j i}-\beta_{j i}\right)=O_{p}(1)$ as $n_{0}$ diverges, for $j=1, \ldots, k, i=1, \ldots, m$;

\footnotetext{
${ }^{1}$ If we call $\boldsymbol{X}_{0}$ and $\boldsymbol{X}_{1}$ the design matrices for the sub-samples $\Omega_{0}$ and $\Omega_{1}$, the variance of the $i$-th regression residual has form $\sigma^{2}\left(1+c_{i}\right)$, where, for $i \in \Omega_{0}, c_{i}$ is the $i$-th element of the diagonal of $-\boldsymbol{X}_{0}\left(\boldsymbol{X}_{0}^{\top} \boldsymbol{X}_{0}\right)^{-1} \boldsymbol{X}_{0}^{\top}$, while for $i \in \Omega_{1}, c_{i}$ is given by the corresponding element on the diagonal of $\boldsymbol{X}_{1}\left(\boldsymbol{X}_{0}^{\top} \boldsymbol{X}_{0}\right)^{-1} \boldsymbol{X}_{1}^{\top}$. Under the usual conditions (and the conditions of Theorem 1) using $\hat{\sigma}^{2}$ or $\hat{\sigma}^{2}\left(1+c_{i}\right)$ for standardising the residuals is asymptotically equivalent, and from our simulations on finite samples, for the sample dimensions used in event studies, there is no practical difference between the two choices.
} 
where $x_{j i t}, \beta_{j i}$ and $\hat{\beta}_{j i}$ are the $j$-th elements of the respective vectors $\boldsymbol{x}_{i t}, \boldsymbol{\beta}_{i}$ and $\hat{\boldsymbol{\beta}}_{i}$.

Then, for the statistic defined in equation (4) it holds

$$
Z_{n} \stackrel{d}{\longrightarrow} N(0,1), \quad \text { as } n_{0} \rightarrow \infty .
$$

The conditions in the theorem are rather mild. Condition 1 requires that the marginal distribution of the sum of the regression residuals has a continuous density which is constant over time; the joint stationarity of the returns and the continuity of their densities are sufficient for this condition. Condition 2 requires the existence of the Fisher Information for location (as in maximum likelihood). Condition 3 asks for a (root- $n$ or faster) consistent estimator for the scale parameter of the regression error ${ }^{2}$. For Condition 4 no single observation of the regressors should (asymptotically) dominate the sum (of squares). Condition 5 allows for any estimator of the regression coefficients that has the same rate of convergence as the OLS estimator ${ }^{3}$ (e.g., LAD, rank, Theil-Sen and other robust estimators can be used instead of OLS).

In order to improve the approximation to the finite-sample distribution of the test statistic we can apply two small corrections to $Z_{n}$. Notice that

$$
T=\sum_{t \in \Omega_{1}} \Phi^{-1}\left(\frac{R_{n t}}{n+1}\right)=\sum_{t=1}^{n} c_{t} a_{R_{n i}}
$$

is a linear rank statistic ${ }^{4}$ with

$$
c_{t}=\left\{\begin{array}{ll}
0 & \text { for } t \in \Omega_{0} \\
1 & \text { for } t \in \Omega_{1}
\end{array}, \quad a_{R_{n t}}=\Phi^{-1}\left(\frac{R_{n t}}{n+1}\right),\right.
$$

\footnotetext{
${ }^{2}$ Actually consistency is not needed, but just convergence in probability to some constant.

${ }^{3}$ Again, the estimator does not have to be consistent, but it is sufficient that it converges in probability to some constant.

${ }^{4}$ The reader may wonder why we do not apply standard linear rank statistic asymptotics to $Z_{n}$. Indeed, this is not possible for two reasons: i) we apply the ranks to estimated regression residuals and not to raw observations, ii) our asymptotic results should work for $n_{0} \rightarrow \infty$ and $n_{1}$ fixed, while for linear rank statistics both sample sizes have to diverge at the same rate.
} 
and, under the null hypothesis, has mean and variance (cf. van der Vaart, 1998, Lemma 13.1)

$$
\mathrm{E} T=n \bar{c} \bar{a}=0, \quad \operatorname{Var} T=\frac{1}{n-1} \sum_{t=1}^{n}\left(c_{t}-\bar{c}\right)^{2} \sum_{t=1}^{n}\left(a_{t}-\bar{a}\right)^{2}=\frac{n_{0} n_{1}}{n} V_{n}
$$

with

$$
V_{n}=\frac{1}{n-1} \sum_{t=1}^{n}\left[\Phi^{-1}\left(\frac{t}{n+1}\right)\right]^{2} .
$$

Of course, as $n \rightarrow \infty$ the variance $V_{n}$ converges to

$$
\int_{0}^{1} \Phi^{-1}(u)^{2} \mathrm{~d} u=\int_{-\infty}^{\infty} x^{2} \phi(x) \mathrm{d} x=1,
$$

where $\phi($.$) denotes the standard normal density function, but, for finite n$, $V_{n}$ tends to be smaller than 1.

If instead of the normalized ranks $R_{n t} /(n+1)$, we use the asymptotically equivalent quantities $\left(R_{n t}-0.5\right) / n$, then the variance $V_{n}$ is very close to 1 also for moderate samples (see Figure 1).

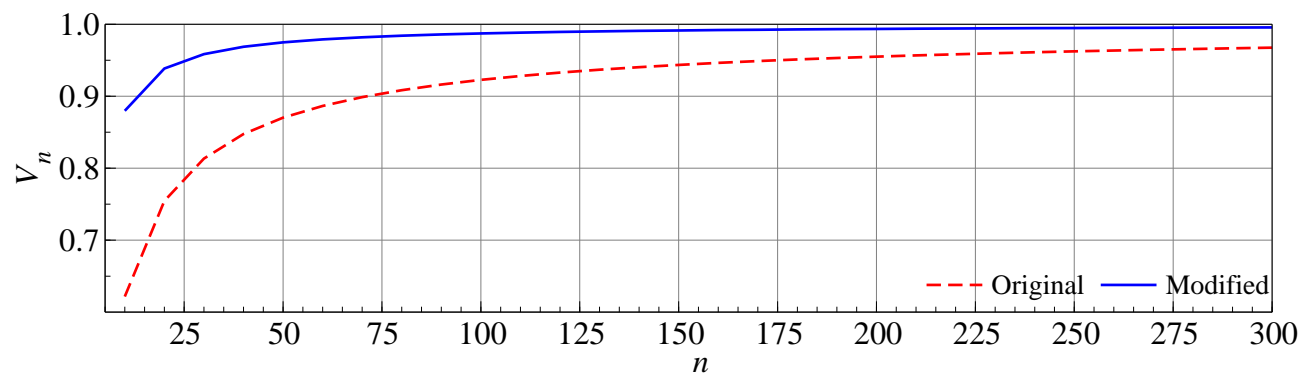

Figure 1: Variance $V_{n}$ as a function of the sample size for the original and modified normalised ranks.

This reasoning suggests the modification of the test statistic (4) with the asymptotically equivalent (as $n_{0} \rightarrow \infty$ )

$$
\tilde{Z}_{n}=\sqrt{\frac{n}{n_{0} n_{1}}} \sum_{t \in \Omega_{1}} \Phi^{-1}\left(\frac{R_{n t}-0.5}{n}\right),
$$


obtained by dividing $T$ by its (approximate) standard deviation $\sqrt{n_{0} n_{1} / n}$.

Simulations (not reported) confirm that the test based on $\tilde{Z}_{n}$ has a more accurate size in small samples then $Z_{n}$.

\section{Alternative tests lacking a solid approxima- tion theory}

It would be interesting to compare the proposed test with alternative tests but, without the assumption of joint normality of the errors $\varepsilon_{t}$ or strong and non realistic restrictions on the dependence among the elements of $\varepsilon_{t}$, it is not easy to build alternative event study tests under cross-sectional dependence.

Thus, in the simulation experiment (see Section 4) we compare size and power of the proposed test with the following three tests, for which a sound asymptotic theory cannot be derived.

Extended Patell (EP) The classical Patell test statistic can be extended to the case of cross-sectional dependence as

$$
E P=\frac{1}{\sqrt{n_{1}}} \sum_{t \in \Omega_{1}} \frac{e_{t}}{\hat{\sigma}_{e}},
$$

where $e_{t}$ has been defied in equation (3) and in the following lines and

$$
\hat{\sigma}_{e}=\left(\sum_{i=1}^{m} \sum_{j=1}^{m} \hat{\sigma}_{i j}^{2}\right)^{1 / 2},
$$

with $\hat{\sigma}_{i j}^{2}=\sum_{t \in \Omega_{0}} e_{i t} e_{j t} /\left(n_{0}-k\right)$, being $k$ the number of regressors in the vector $\boldsymbol{x}_{i t}$.

Under the hypothesis of joint normality of $\varepsilon_{t}$ for all $t$, as $n_{0}$ diverges, the sample covariances converge almost surely to the corresponding population moments and EP converges in distribution to a standard normal. Without the joint normality assumption, an asymptotic distribution theory is not available. 
Extended Corrado (EC) The popular rank test proposed by Corrado (1989) can be extended to the cross-sectionally dependent case as

$$
E C=\sqrt{\frac{12}{n_{1}}} \sum_{t \in \Omega_{1}} \sum_{i=1}^{m} \frac{U_{i t}-1 / 2}{\hat{\varrho}^{1 / 2}},
$$

where $U_{i t}=R_{n i t} /(n+1)$, being $R_{n i t}$ the rank of $e_{i t}$ among $\left\{e_{i 1}, \ldots, e_{i n}\right\}$ and

$$
\hat{\varrho}=\sum_{i=1}^{m} \sum_{j=1}^{m} \hat{\varrho}_{i j},
$$

with $\hat{\varrho}_{i j}$ Spearman's rank correlation between $e_{i t}$ and $e_{j t}$ based on the sample $\Omega_{0}$.

Now, if there is only one stock $(m=1)$ and $n_{1}$ is moderately large the standard normal turns out to be a good approximation to the actual distribution. Unfortunately, if $m>1$ under general cross sectional dependence we cannot relay on any central limit theorem (CLT) to justify the normal approximation. Indeed, CLTs for dependent variables were developed for stochastic processes, thus, with an ordered indexing of the random variables. Some form of mixing and the existence of higher order moments are the usual assumptions under which the CLT holds. While $U_{i t}$ possesses moments of any order (i.e., it is a bounded random variable), it is not possible to apply the concept of mixing in a meaningful way. To the best of our knowledge the only conditions that could be adapted to the Extended Corrado statistic are those of McLeish (1974, Theorem 2.1), but they are not easy to verify.

Van der Waerden scores (WS) In order to accelerate the (eventual) convergence of the Corrado statistic to the standard normal distribution, we can apply van der Waerden type scores (van der Waerden, 1952) to the ranks. The test statistic is

$$
W S=\frac{1}{\sqrt{n_{1}}} \sum_{t \in \Omega_{1}} \sum_{i=1}^{m} \frac{\Phi^{-1}\left(U_{i t}\right)}{\hat{\rho}^{1 / 2}},
$$

where

$$
\hat{\rho}=\sum_{i=1}^{m} \sum_{j=1}^{m} \hat{\rho}_{i j}
$$


with $\hat{\rho}_{i j}$ sample correlation between $\Phi^{-1}\left(U_{i t}\right)$ and $\Phi^{-1}\left(U_{j t}\right)$ computed using the sub-sample $\Omega_{0}$.

For $m=1$ the test statistic WS is identical to $Z$ of Equation (4) and converges in distribution to the standard normal as $n_{0}$ diverges, regardless of the size of $n_{1}$. For $m>1$ the same CLT-related considerations as for the $E C$ statistic hold, but for the distribution of WS the normal approximation should be more accurate for small $m$ since we are summing (dependent) approximately normal random variables instead of (dependent) uniform random variables.

Notice that all the considered tests $(Z, E P, E C, W S)$ can be easily modified to include the adjustment proposed by Boehmer et al. (1991) (see also Kolari and Pynnönen, 2010) that makes the tests robust to event-induced volatility and that of Kolari and Pynnönen (2011), which increases the power of the tests when only a small portion of the returns in the event window has a shifted distribution.

\section{Simulation experiment}

The finite sample behaviour of the four tests is investigated by bootstrapping the daily returns of stocks selected from the S\&P100 and NASDAQ indexes. The daily returns were recorded over the period February 2003-February 2013 (S\&P100) and April 2003-April 2013 (NASDAQ).

The estimation window is fixed at $n_{0}=250$ observations and two event windows of $n_{1}=1$ and $n_{1}=5$ days are considered. We apply the tests to different numbers of stocks: $m=\{5,10,20,40,80\}$. The stocks are chosen according to a maximum correlation criterion and, therefore, the average correlation in each set of stocks decreases with $m$.

The tests are applied both to the raw returns (after subtracting the mean for the EP test) and to the residuals of the (OLS) regression of the stock returns on their respective indexes (market model).

Tables 1 and 2 report the actual sizes of the tests for a nominal size of $5 \%$. The test $Z$ has the most accurate size (range $4.7 \%-5.2 \%$ ) and its size properties are very similar regardless of the set-up. As expected, the $E C$ test has serious size problems (especially for NASDAQ stocks) when the number of securities $m$ is small and the average correlation is high (range 
Table 1: Nominal sizes of the four tests for an actual 5\% size: S\&P100 stocks.

\begin{tabular}{|c|c|c|c|c|c|c|c|c|c|c|}
\hline \multirow{3}{*}{$\begin{array}{l}\text { N. stocks } \\
\text { Mean corr. }\end{array}$} & \multicolumn{5}{|c|}{ Raw returns } & \multicolumn{5}{|c|}{ Market model residuals } \\
\hline & 5 & 10 & 20 & 40 & 80 & 5 & 10 & 20 & 40 & 80 \\
\hline & 64 & .60 & .57 & .53 & .47 & 27 & .18 & .14 & .04 & .00 \\
\hline \multicolumn{11}{|l|}{$n_{1}=1$} \\
\hline Z & 4.8 & 4.8 & 4.8 & 4.7 & 4.9 & 4.9 & 4.9 & 5.2 & 4.9 & 5.2 \\
\hline WS & 7 & 5.9 & 5.8 & 6.2 & 6.5 & 7 & 5.0 & 5.8 & 5.3 & 5.1 \\
\hline EC & .6 & 4.5 & 4.9 & 5.5 & 6.0 & 3.4 & 4.4 & 4.4 & 5.1 & 4.9 \\
\hline EP & 5.0 & 5.0 & 5.0 & 5.3 & 5.7 & 6.2 & 5.6 & 6.2 & 5.7 & 5.5 \\
\hline \multicolumn{11}{|l|}{$n_{1}=5$} \\
\hline $\mathrm{Z}$ & 4.9 & 5.1 & 5.0 & 5.0 & 4.9 & 5.1 & 5.2 & 5.2 & 5.1 & 5.2 \\
\hline WS & 4.8 & 4.9 & 5.0 & 5.1 & 5.1 & 4.9 & 5.0 & 5.0 & 5.0 & 5.3 \\
\hline EC & 4.5 & 4.5 & 4.6 & 4.7 & 4.7 & 4.7 & 4.7 & 4.9 & 4.7 & 5.0 \\
\hline EP & 5.9 & 6.7 & 6.3 & 6.4 & 6.3 & 5.9 & 6.3 & 6.2 & 6.0 & 6.4 \\
\hline
\end{tabular}

$0.2 \%-6.0 \%)$. The parametric test $E P$ and the nonparametric $W S$ tend to have good size properties.

Figures 2-5 depict the powers of the four tests under the considered setups. The drift in the mean in the event window is expressed in number of standard deviations, as all the stock returns received a drift proportional to their respective standard deviations.

From Figure 2, it appears that under highly correlated returns, when the number of stocks is small and the event window coincides with the event date $\left(n_{1}=1\right)$ the size problems of the $E C$ test are reflected in its power, but as soon as the number of stocks increases to 20 or more, the $E C$ becomes one of the most powerful tests in the analysis. In all the other set-ups the $E C$ performs extremely well.

The $W S$ test tends to have a competitive power under all conditions: it behaves similarly to the $E C$ when this test performs well, without suffering from its problems when the correlation is high and $n_{1}=1$.

The $Z$ test and the parametric $E P$ test tend to have less power than the two competitors and none of the two is uniformly better than the other. However, the $E P$ is always slightly oversized (i.e., it rejects too often) and this increases its power artificially. It is also likely that under conditions of 
Table 2: Nominal sizes of the four tests for an actual 5\% size: NASDAQ stocks.

\begin{tabular}{|c|c|c|c|c|c|c|c|c|c|c|}
\hline \multirow[b]{2}{*}{ N. of stocks } & \multicolumn{5}{|c|}{ Raw returns } & \multicolumn{5}{|c|}{ Market model residuals } \\
\hline & 5 & 10 & 20 & 40 & 80 & 5 & 10 & 20 & 40 & 80 \\
\hline Mean corr. & .71 & .61 & .53 & .49 & .39 & 41 & .24 & .12 & .06 & .03 \\
\hline \multicolumn{11}{|l|}{$n_{1}=1$} \\
\hline $\mathrm{Z}$ & 4.8 & 4.7 & 4.9 & 4.8 & 4.8 & 4.9 & 5.1 & 5.1 & 5.0 & 5.2 \\
\hline WS & 5.1 & 5.2 & 5.9 & 6.1 & 6.1 & 5.5 & 5.4 & 5.8 & 5.7 & 5.0 \\
\hline EC & 0.2 & 1.9 & 4.0 & 4.7 & 5.0 & 3.6 & 4.3 & 5.3 & 5.7 & 5.0 \\
\hline EP & 5.9 & 5.6 & 6.0 & 5.8 & 5.6 & 6.2 & 6.0 & 6.1 & 6.0 & 5.2 \\
\hline \multicolumn{11}{|l|}{$n_{1}=5$} \\
\hline $\mathrm{Z}$ & 4.8 & 5.1 & 5.0 & 5.1 & 5.0 & 5.1 & 5.1 & 5.3 & 5.4 & 5.1 \\
\hline WS & 4.6 & 4.9 & 4.8 & 5.0 & 4.8 & 5.0 & 4.9 & 5.2 & 5.4 & 5.2 \\
\hline EC & 4.4 & 4.7 & 4.7 & 4.8 & 4.6 & 4.7 & 4.7 & 5.0 & 5.2 & 5.0 \\
\hline EP & 5.5 & 5.7 & 5.7 & 5.9 & 5.7 & 6.0 & 5.9 & 6.0 & 6.2 & 5.9 \\
\hline
\end{tabular}

extreme market movements the size of the $E P$ test will farther inflate, while those of the other three statistics will remain unaffected, being distributionfree tests.

Thus, the WS test seems to be a good alternative to the $E C$ that does not suffer from size problem but preserves the same good power features. The $Z$ test has power properties that are comparable to standard EC test, but being endowed with a solid distribution theory also under non-normality, it is more likely to provide sensible results in any possible set-up.

\section{Conclusion}

In this paper we proposed three new nonparametric tests for the null of no event-induced shift in the distribution of stock returns and compared them with the well known Patell test $(E P)$. One of these test statistic $(Z)$ is distribution free and comes with a sound asymptotic theory that permits the accurate approximation of its distribution under typical set-ups. The other two tests ( $E C$ and $W S$ ) are not supported by an approximate distribution theory and their properties are assessed only by simulation experiments.

The $Z$ test keeps its promise in terms of size, but its power is compara- 

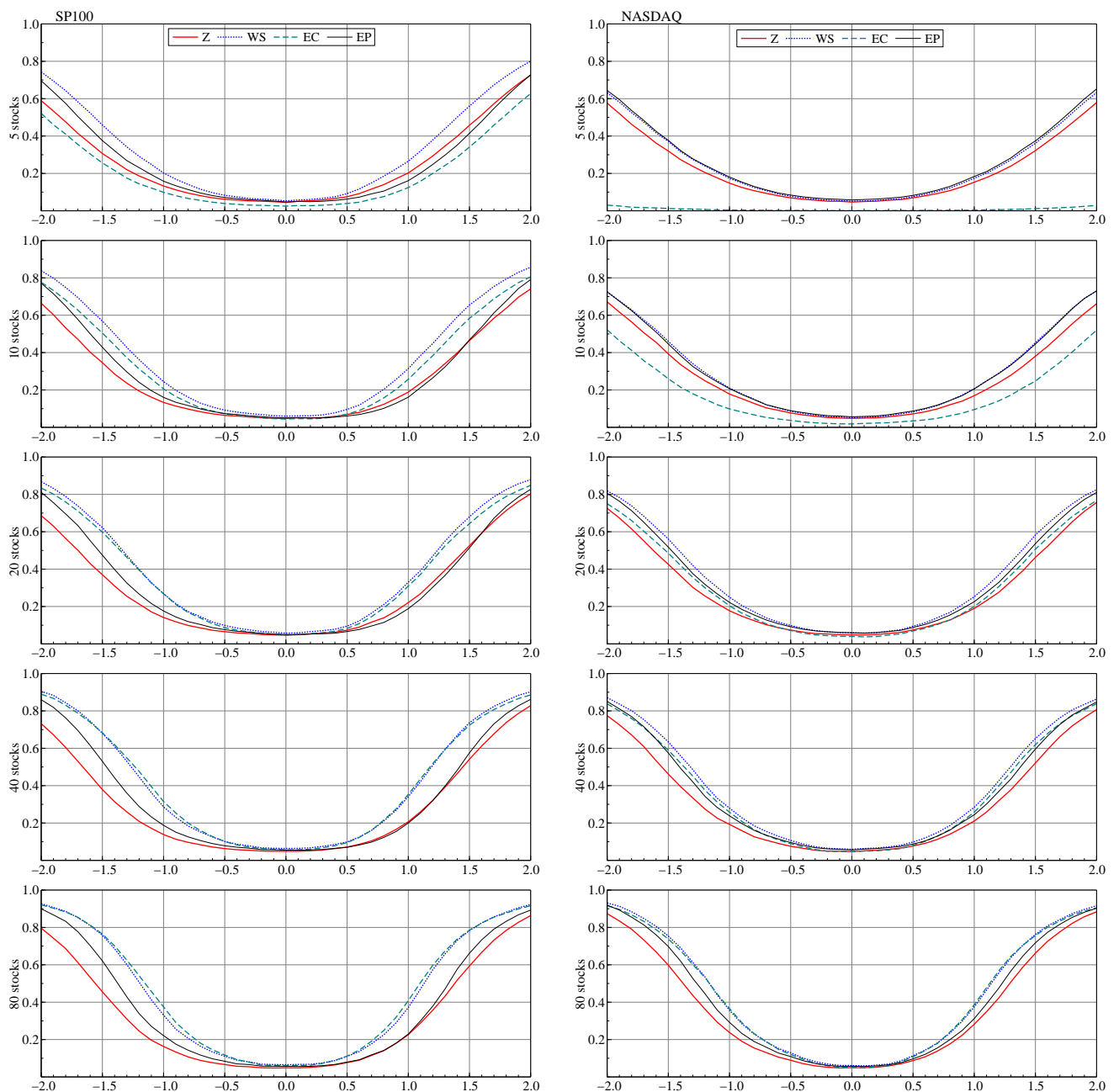

Figure 2: Power of the tests computed on raw returns taken from the SP100 and NASDAQ most correlated constituents for an event windows of $n_{1}=1$ days. 

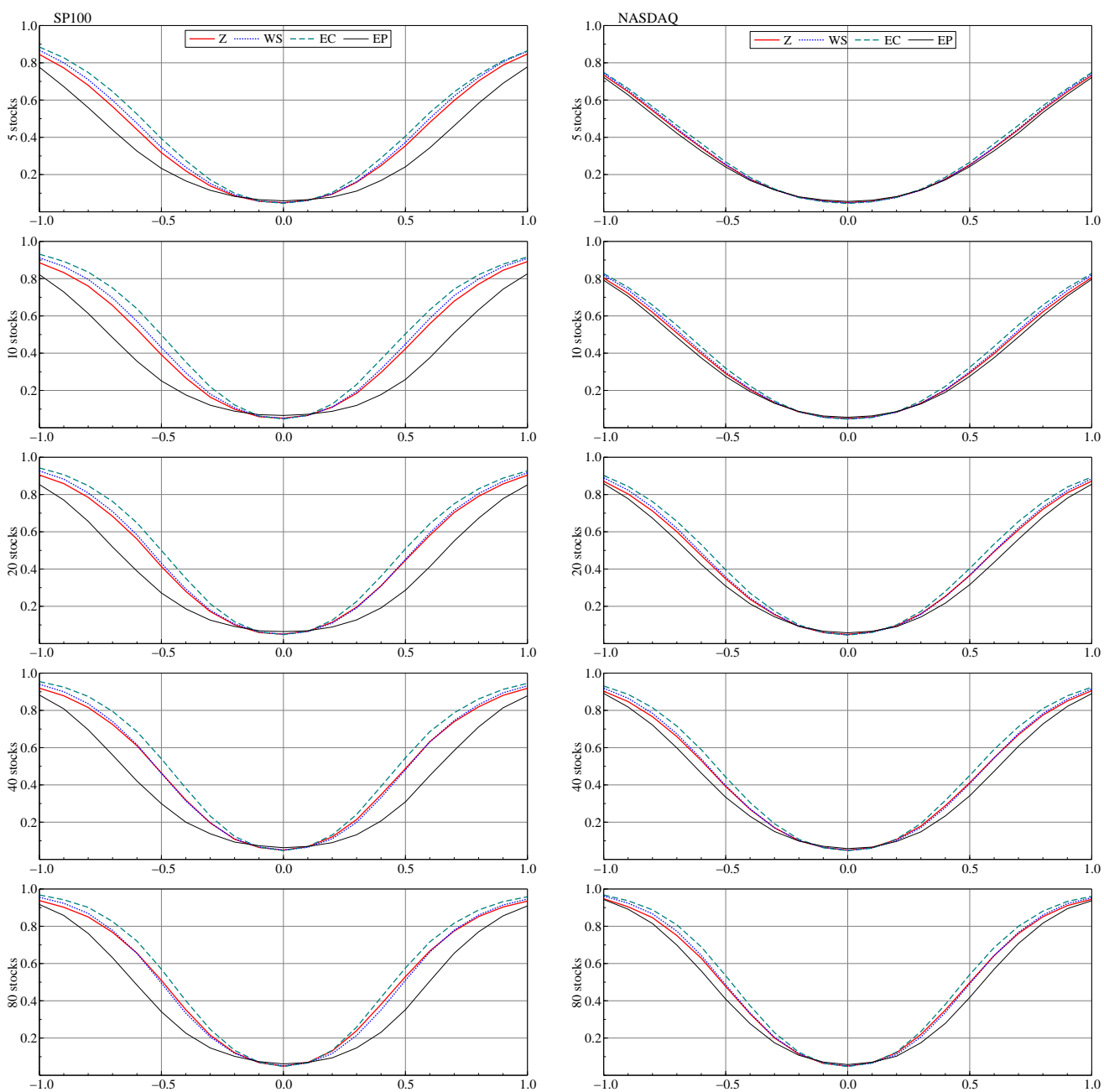

Figure 3: Power of the tests computed on raw returns taken from the SP100 and NASDAQ most correlated constituents for an event windows of $n_{1}=5$ days. 

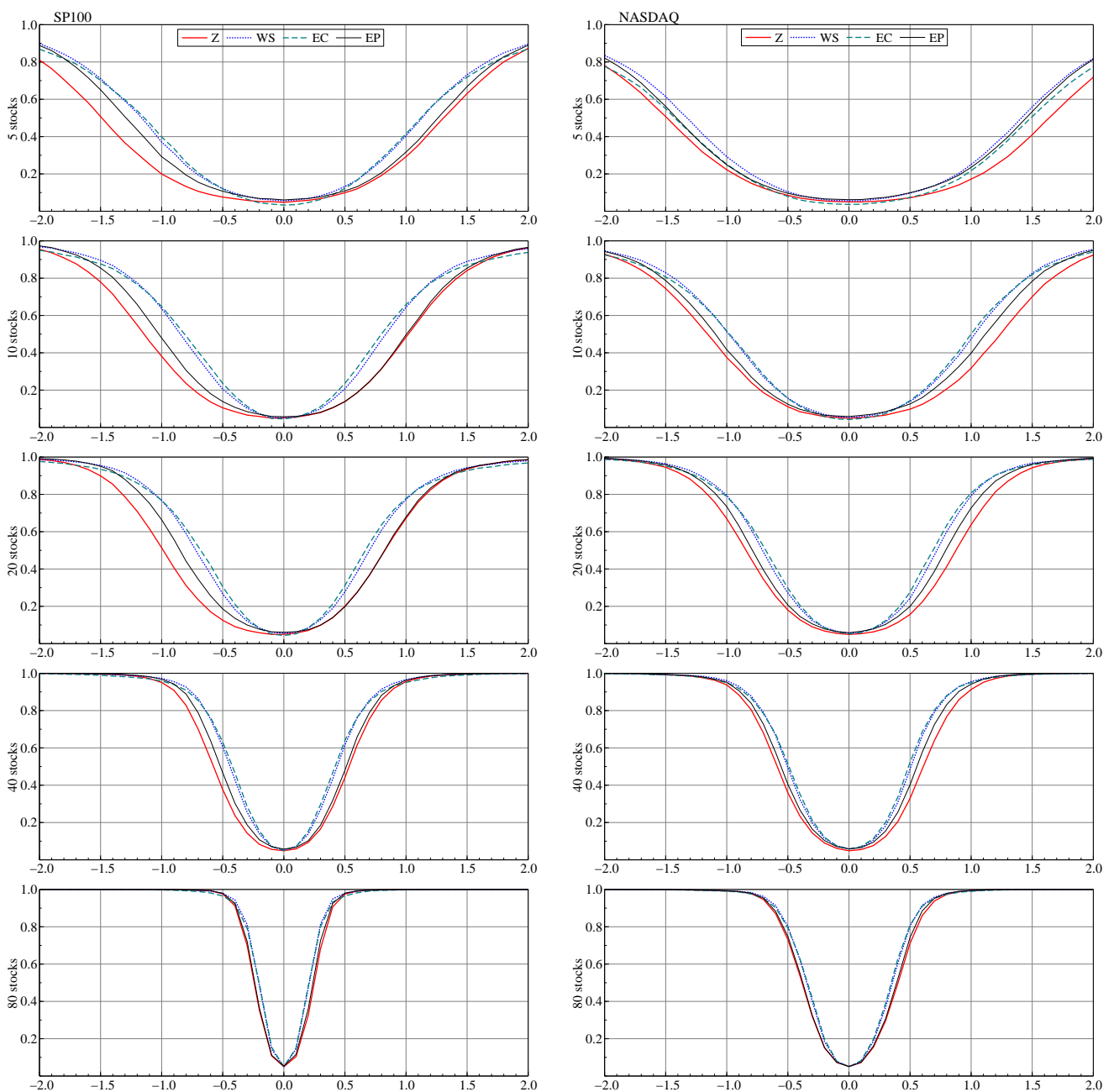

Figure 4: Power of the tests computed on market model residuals taken from the SP100 and NASDAQ most correlated constituents for an event windows of $n_{1}=1$ days. 

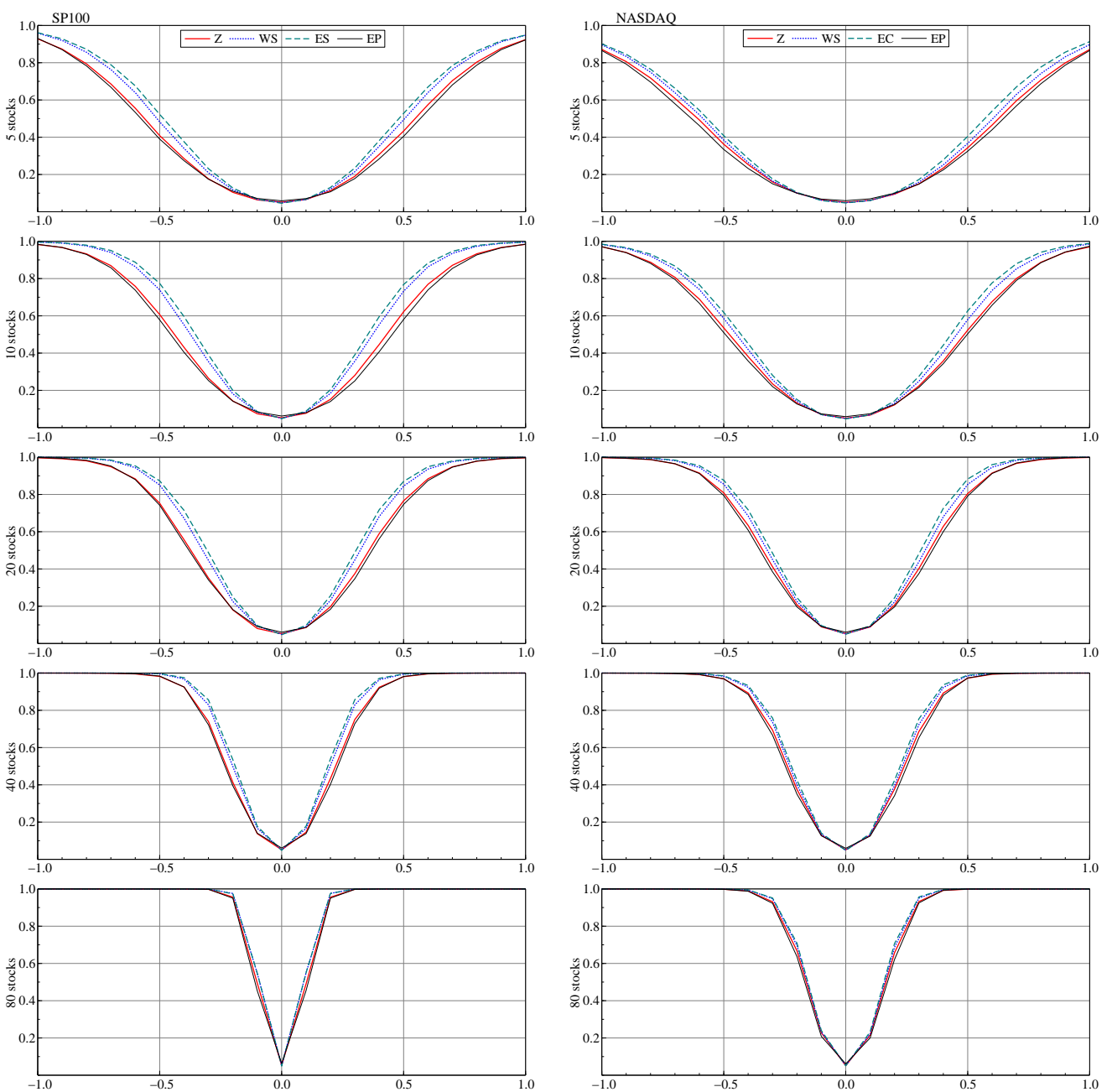

Figure 5: Power of the tests computed on market model residuals taken from the SP100 and NASDAQ most correlated constituents for an event windows of $n_{1}=5$ days. 
ble to that of the (extended) Patell test (EP), which, however, is somewhat oversized. The rank test based on van der Vaart scores (WS) and the extended Corrado statistic perform better in terms of power, but the latter has serious size problems when the number of stocks is small and the event window short.

Thus, the simulation results suggest the use of the $W S$ test, even though a solid approximate distribution theory is missing. However, in practical implementations the lack of theory can represent e serious problem unless results are supported by simulations like the ones in this paper that confirm the size under the null for the dataset under analysis. On the contrary, the $Z$ statistic is always safe as the approximate distribution is known under extremely mild regularity conditions and the size is excellent under any distribution of the asset returns that possesses a continuous density. We think that our $Z$ statistic should replace the widely used Patell T-test in any event study (univaritate or multivariate) as, for the typical distribution of financial asset returns, it gains in robustness without loosing any power.

A comparison of the same tests modified as suggested by Boehmer et al. (1991), Kolari and Pynnönen (2010) and Kolari and Pynnönen (2011) will be the natural follow-up of this paper.

\section{References}

Bartholdy, J., D. Olson, and P. Peare (2007). Conducting event studies on a small stock exchange. The European Journal of Finance 13(3), 227-252.

Boehmer, E., J. Musumeci, and A. B. Poulsen (1991). Event-study methodology under conditions of event-induced variance. Journal of Financial Economics 30(2), 253-272.

Corrado, C. J. (1989). A nonparametric test for abnormal security-price performance in event studies. Journal of Financial Economics 23, 385395.

Corrado, C. J. and C. Truong (2008). Conducting event studies with asiapacific security market data. Pacific-Basin Finance Journal 16, 493-521.

Corrado, C. J. and T. L. Zivney (1992). The specification and power of the sign test in event study hypothesis tests using daily stock returns. Journal of Financial and Quantitative Analysis 27(3), 465-478. 
Dodd, P. and J. B. Warner (1983). On corporate governance: a study of proxy contests. Journal of Financial Economics 11(1), 401. 11(1), 401438.

Kolari, J. W. and S. Pynnönen (2010). Event study testing with crosssectional correlation of abnormal returns. Review of Financial Studies 23(11), 3996-4025.

Kolari, J. W. and S. Pynnönen (2011). Nonparametric rank tests for event studies. Journal of Empirical Finance 18(5), 953-971.

Lummer, S. and J. McConnell (1989). Further evidence on the bank lending process and the capital market response to bank loan agreements. Journal of Financial Economics 25(1), 88-122.

McConnell, J. and C. Muscarella (1985). Corporate capital expenditure decisions and the market value of the firm. Journal of Financial Economics 14(3), 399-422.

McLeish, D. L. (1974). Dependent central limit theorems and invariance principles. Annals of Probability 2(4), 620-628.

Patell, J. M. (1976). Corporate forecasts of earnings per share and stock price behavior: empirical tests. Journal of Accounting Research 14, 246 276.

Sanders, R. W. J. and R. P. Robins (1991). Discriminating between wealth and information effects in event studies in accounting and finance research. Review of Quantitative Finance and Accounting 1(3), 307-330.

van der Vaart, A. W. (1998). Asymptotic Statistics. Cambridge series in statistical and probablistic mathematics. The Edinburgh Building, Cambridge CB2 2RU, UK: Cambridge University Press.

van der Waerden, B. (1952). Order tests for the two-sample problem and their power. Indagationes Mathematicae 14, 453-458.

Zivney, T. L. and D. J. Thompson (1989). The specification and power of the sign test in measuring security price performance: comments and analysis. The Financial Review 24, 581-588. 


\section{Appendix}

Proof of Theorem 1. Let $F_{n}(x)=n^{-1} \sum_{t \in \Omega} \mathbb{1}_{(-\infty, x]}\left(\varepsilon_{t}\right)$ be the empirical distribution function of $\varepsilon_{t}$. By standard empirical process theory, $F_{n}(x)=$ $F(x)+O_{p}\left(n^{-1 / 2}\right)$ uniformly in $x$.

By Taylor expansion of $\hat{\sigma}_{i}^{-1}$ in a neighbourhood of $\sigma_{i}$,

$$
\begin{aligned}
\frac{\hat{\varepsilon}_{i t}}{\hat{\sigma}_{i}} & =\frac{\varepsilon_{i t}}{\hat{\sigma}_{i}}-\frac{\boldsymbol{x}_{i t}^{\top}\left(\hat{\beta}_{i}-\beta\right)}{\hat{\sigma}_{i}} \\
& =\frac{\varepsilon_{i t}}{\sigma_{i}}+\frac{\varepsilon_{i t}}{\sigma_{i}^{2}}\left(\hat{\sigma}_{i}-\sigma_{i}\right)-\frac{\boldsymbol{x}_{i t}^{\top}\left(\hat{\beta}_{i}-\beta\right)}{\hat{\sigma}_{i}}+o_{p}\left(n^{-1 / 2}\right) \\
& =\frac{\varepsilon_{i t}}{\sigma_{i}}+\delta_{n i},
\end{aligned}
$$

where $\delta_{n i}$ is equal to the last three addends of the second line of the above equations and, by assumptions 3., 4., 5., $\delta_{n i}=O_{p}\left(n^{-1 / 2}\right)$.

Then,

$$
e_{t}=\sum_{i=i}^{m} \frac{\hat{\varepsilon}_{i t}}{\hat{\sigma}_{i}}=\sum_{i=i}^{m}\left(\frac{\varepsilon_{i t}}{\sigma}+\delta_{n i}\right)=\varepsilon_{t}+\delta_{n},
$$

where $\delta_{n}=\sum_{i=1}^{m} \delta_{n i}=O_{p}\left(n^{-1 / 2}\right)$. By Taylor's theorem, for some $h_{\varepsilon, \delta} \in$ $[0,1]$

$$
F\left(e_{t}\right)-F\left(\varepsilon_{t}\right)-f\left(\varepsilon_{t}\right) \delta_{n}=f\left(\varepsilon_{t}+h_{\varepsilon, \delta} \delta_{n}\right)-f\left(\varepsilon_{t}\right)
$$

For any $\varepsilon$ and $\delta$, by applying the Cauchy-Schwarz inequality we can write

$$
\begin{aligned}
|f(\varepsilon+h \delta)-f(\varepsilon)| & =\left|\int_{0}^{h \delta} f^{\prime}(\varepsilon+u) \mathrm{d} u\right| \leq \int_{0}^{\delta}\left|f^{\prime}(\varepsilon+u)\right| \mathrm{d} u \\
& =\int_{0}^{\delta} \frac{\left|f^{\prime}(\varepsilon+u)\right|}{\sqrt{f(\varepsilon+u)}} \sqrt{f(\varepsilon+u)} \mathrm{d} u \\
& \leq\left[\int_{0}^{\delta}\left(\frac{f^{\prime}(\varepsilon+u)}{f(\varepsilon+u)}\right)^{2} f(\varepsilon+u) \mathrm{d} u \cdot \int_{0}^{\delta} f(\varepsilon+u) \mathrm{d} u\right]^{\frac{1}{2}} \\
& \leq\left[\int_{0}^{\delta}\left(\frac{f^{\prime}(\varepsilon+u)}{f(\varepsilon+u)}\right)^{2} f(\varepsilon+u) \mathrm{d} u \cdot[F(\varepsilon+\delta)-F(\varepsilon)]\right]^{\frac{1}{2}} \\
& \leq \sqrt{I_{0}(f)} \cdot \sqrt{F(\varepsilon+\delta)-F(\varepsilon)},
\end{aligned}
$$


which by assumptions 1 . and 2 . is bounded. Thus, we have

$$
F\left(e_{t}\right)=F\left(\varepsilon_{t}\right)+O_{p}\left(n^{-1 / 2}\right) .
$$

Putting the pieces together we have

$$
U_{n t}=\frac{R_{n t}}{n+1}=\frac{n+1}{n} F_{n}\left(e_{t}\right)=F\left(e_{t}\right)+O_{p}\left(n^{-1 / 2}\right)=F\left(\varepsilon_{t}\right)+O_{p}\left(n^{-1 / 2}\right),
$$

which converges in probability to a uniform random variable, say $U_{t}$. Thus, by the probability integral transform, as $n_{0} \rightarrow \infty$

$$
\Phi^{-1}\left(U_{n t}\right) \stackrel{p}{\longrightarrow} \Phi^{-1}\left(U_{t}\right) \sim N(0,1) .
$$

Moreover, as $n_{0}$ diverges ranks become independent and, thus, for fixed $n_{1}$

$$
\sum_{t \in \Omega_{1}} \Phi^{-1}\left(U_{n t}\right) \stackrel{p}{\longrightarrow} \sum_{t \in \Omega_{1}} \Phi^{-1}\left(U_{t}\right) \quad \text { as } n_{0} \rightarrow \infty
$$

which is the sum of $n_{1}$ independent standard normal random variables. 